\title{
Effect of inhibitors of endocytosis and NF-kB signal pathway on folate-conjugated nanoparticle endocytosis by rat Kupffer cells
}

This article was published in the following Dove Press journal:

International Journal of Nanomedicine

18 September 2017

Number of times this article has been viewed

\author{
Hongbo Tang' \\ Hongli Chen ${ }^{2}$ \\ Yajing Jia ${ }^{2}$ \\ Xiaoyan Liu' \\ Zhaohong Han' \\ Aihua Wang' \\ Qi Liu' \\ Xinlei Li' \\ Xin Feng' \\ 'Department of Pharmacy, Beijing \\ Obstetrics and Gynecology \\ Hospital, Capital Medical University, \\ Beijing, ${ }^{2}$ School of Life Sciences \\ and Technology, Xinxiang Medical \\ University, Xinxiang, Henan, People's \\ Republic of China
}

Correspondence: Xin Feng

Department of Pharmacy, Beijing

Obstetrics and Gynecology Hospital,

Capital Medical University, No 17,

Qi He Lou Street, Dongcheng District,

Beijing 100006, People's Republic

of China

Tel +86 I0 5227303 I

Fax +86 I0 85968425

Email fengxinIII5@I26.com
Abstract: The regular accumulation of nanoparticles in the liver makes them hepatotoxic and decreases the circulation time, thus reducing their therapeutic effect. Resolving this problem will be significant in improving bioavailability and reducing side effects. In this study, we reduced the phagocytosis of epirubicin (EPI)-loaded folic acid-conjugated pullulan acetate (FPA/ EPI) nanoparticles by Kupffer cells (KCs) through internalization and nuclear factor kappa B (NF-kB) signal pathway inhibitors, thus allowing development of FPA/EPI nanoparticles as a nanodrug delivery system (NDDS) based on our previous study. FPA/EPI nanoparticles were prepared by the dialysis method. Rat KCs were preincubated with the following individual or compound inhibitors: chlorpromazine (CPZ), nystatin (NY), colchicine (Col), amiloride (AMR), and pyrrolidine dithiocarbamate (PDTC). Dose- and time-dependent cellular uptake effects of inhibitors on FPA/EPI nanoparticles were determined through fluorometry. The cytokine levels of tumor necrosis factor alpha (TNF- $\alpha$ ), interleukin-1 beta (IL-1 $\beta$ ), and IL-6 were tested in culture supernatants by bead-based multiplex flow cytometry. The uptake study demonstrated that inhibitors had an obvious inhibitory effect $(P<0.05$ or $P<0.01)$, with NY, AMR and Col all showing time-dependent inhibitory effects. PDTC $+\mathrm{NY}$ had the strongest inhibitory effect, with an uptake rate of $14.62 \%$. The levels of the three proinflammatory cytokines were changed significantly by the compound inhibitors. TNF- $\alpha$ was significantly inhibited $(P<0.05$ or $P<0.01)$, but IL-1 $\beta$ and IL-6 showed smaller decreases. These results suggested that clathrin- and caveolae-mediated endocytosis were the main routes via which nanoparticles entered KCs and that the NF-kB signal pathway was very important too. In summary, multiple mechanisms, including clathrin- and caveolae-mediated endocytosis, contribute to cytokine production in macrophages following exposure to folic acid-conjugated pullulan acetate nanoparticles. Thus, the endocytosis inhibition strategy has great potential for improving therapy and reducing toxicity of an NDDS in the treatment of cancer.

Keywords: nanodrug delivery system, Kupffer cells, endocytosis inhibitor, folate-conjugated pullulan acetate, NF-kB signal pathway

\section{Introduction}

Nanomedicine is a new field being developed in the 21 st century. Polymeric micelles, quantum dots, liposomes, polymer-drug conjugates, dendrimers, biodegradable nanoparticles (NPs), silica NPs, and so on are classic examples of nanoparticulate materials currently being researched in laboratories, undergoing preclinical development, or already used in the clinic. ${ }^{1}$ The internalization of these polymeric NPs into cells depends on several variables, including their size, surface properties, and cell type. The reticuloendothelial system composed of the liver and spleen is the first and most 
urgent immunological barrier to intravenously administered NPs, as it can remove the NPs from the circulation. ${ }^{2}$ Kupffer cells (KCs) are the largest population of fixed macrophages in the body and contribute to hepatic uptake of both small and large microspheres. Some results suggested that at least immediately after administration, uptake into the liver occurs primarily via KCs rather than via parenchymal or endothelial cells. ${ }^{3,4}$ Morais et $\mathrm{al}^{5}$ evaluated the biodistribution of $20 \mathrm{~nm}$ gold nanoparticles (GNPs) with six different surface coatings and demonstrated that GNPs are rapidly distributed, while liver is the preferential accumulation organ, with GNPs trapped in KCs, hepatocytes, and endosomes. Sadauskas et $\mathrm{al}^{6}$ showed that GNPs, independent of size (2 and $40 \mathrm{~nm}$ ), were taken up primarily by KCs in the liver and secondarily by macrophages at other sites. ${ }^{7}$

NPs cannot simply enter cells via diffusion. The pathways of uptake of NPs can be divided into phagocytosis/ macropinocytosis, receptor-mediated endocytosis, and passive penetration. Their regular accumulation in the liver makes NPs ideal candidates for treating hepatic diseases upon parenteral administration; however, it also puts them into focus due to the liver toxicity of therapeutic strategies that do not intend to target the liver as well as the decreased circulation time, which reduces the pharmacological effect. This is an urgent problem with the use of NPs. ${ }^{8}$ Resolving the problem will significantly improve the bioavailability of targeted nanodrugs, enhancing the delivery of the drug to the targeted lesion site, thus improving its therapeutic effect and reducing side effects. ${ }^{3,9}$ The surface modification of NPs by polyethylene glycol (PEG) was developed as the first strategy to prolong NP circulation. While PEGylation has helped prolong particle circulation time, it has several limitations, including the transient nature of the effect and the compromised particle-target interactions. Accordingly, several other approaches have been developed to prolong NP circulation in the blood. These include modification with poly(ethylene oxide), dextran, polyacrylic acid, protein- and peptide-based targeting molecules, folate (FA)-based targeting molecules, carbohydrate-based targeting molecules, and monoclonal antibodies, among others. ${ }^{10,11}$ Another important strategy is immunity inhibition. Wang et al ${ }^{12}$ characterized the internalization of NPs using pharmacological inhibitors and found indications of two coexisting uptake mechanisms: actin- and clathrin-dependent endocytosis. Nuclear factor kappa $\mathrm{B}(\mathrm{NF}-\mathrm{kB})$ may regulate phagocyte activation via the induction of cytokine production. ${ }^{13}$ Khan et $\mathrm{al}^{14}$ studied the time course of the effects of 10 and $50 \mathrm{~nm}$ diameter naked GNPs on the expression of proinflammatory cytokines, including interleukin (IL)-1 beta (IL-1 $\beta$ ), IL-6, and tumor necrosis factor alpha (TNF- $\alpha$ ), in the liver and kidneys of rats. In the liver, the GNPs of both sizes (10 and $50 \mathrm{~nm}$ ) significantly increased cytokine gene expression on Day 1 , which subsided by Day 5; however, the $50 \mathrm{~nm}$ GNPs produced a more severe inflammatory response compared to the $10 \mathrm{~nm}$ GNPs. Moreover, IL- $1 \alpha$ and IL- $1 \beta$ are likely components of the NF-kB signaling pathway, which can be induced by NF-kB activation. IL- $1 \beta$ is an important proinflammatory cytokine that activates monocytes, macrophages, and neutrophils. ${ }^{15}$ IL-1, IL-6, and TNF- $\alpha$ are very important cytokines affecting the NF-kB signal pathway.

FA is a water-soluble B vitamin, also known as vitamin $\mathrm{B}_{9}$. In adult tissues, FA is mainly transported by the reduced FA carrier and the FA receptors (FRs). ${ }^{16}$ FRs are overexpressed in numerous cancers, such as tumors of the ovary, pediatric ependymal brain, mesothelium, breast, colon, kidney, and lung, as well as head and neck carcinomas, but expression is low or nonexistent in most normal tissues, except the kidneys, lungs, and placenta. ${ }^{16-18}$ Thus, FRs are considered an efficient target for cancer therapy. FA was selected as the targeting ligand since it is one of the bestcharacterized ligands to be exploited for targeting cancer cells. ${ }^{19}$ Many drug delivery vehicles, such as liposomes, proteins, polymeric NPs, linear polymers, and dendrimers, have been modified with FA to increase their cellular uptake in FR-overexpressing tumor cells. ${ }^{16-18}$ Epirubicin (EPI)-loaded FA-conjugated pullulan acetate (FPA/EPI) NPs and FAconjugated chitosan-coated poly(D,L-lactide-co-glycolide) NPs might have potential applications in cervical cancer therapy. ${ }^{20-22}$

Pullulan is a nonimmunogenic, nontoxic, noncarcinogenic, and nonmutagenic material that can be derivatized by chemical reactions with the hydroxyl groups of pyranose rings; therefore, it can be used for targeting drugs and gene delivery. ${ }^{23}$ We have studied several characteristics of EPIloaded FA-conjugated pullulan acetate (FPA/EPI) NPs. ${ }^{20,21}$ FA was conjugated to pullulan acetate to form the conjugated ligand and thereafter the pullulan acetate nanocarrier. Chen et $\mathrm{al}^{18}$ and Kim et $\mathrm{al}^{19}$ revealed that enhanced intracellular uptake by HeLa cells (FR-positive) in vitro was enabled by receptor-mediated endocytosis and that FA contributed to this effect. Our previous in vitro and in vivo results ${ }^{20,21}$ suggested that FPA/EPI NPs showed promise in treating cervical carcinoma because of its high stability, low toxicity, and slow-release pharmacokinetic characteristics. A tissue distribution study showed that FPA/EPI NPs accumulated in the liver and spleen of nude mice for up to $48 \mathrm{~h}$ after a single intravenous (iv) injection. ${ }^{20}$ Therefore, the aim of our study was to determine which internalization mechanism 
is important in enabling cellular uptake of FPA/EPI NPs by macrophages. Using macrophages treated with NPs at a concentration that does not induce cell death, production of the proinflammatory cytokines TNF- $\alpha$, IL-1 $\beta$, and IL- 6 was examined. The dose- and time-dependent effects of inhibitors through the NF-kB signaling and endocytosis pathways were observed. In this investigation, we studied the effects of inhibitors of the NF-kB signaling pathway and an endocytosis reagent, which are expected to reduce the endocytosis of FPA/EPI NPs by KCs, thus prolonging the circulation time and improving the targeted antitumor effects.

\section{Materials and methods Materials}

Pullulan (molecular weight $=200,000 \mathrm{Da}$ ) and EPI $\cdot \mathrm{HCl}$ were purchased from Hayashibara Co, Ltd (Tokyo, Japan) and Hisun Pharmaceutical Co (Zhejiang, China), respectively. RPMI-1640 medium, fetal bovine serum (FBS) (Thermo Fisher Scientific, Waltham, MA, USA), penicillinstreptomycin (pen-strep), and $0.25 \%$ trypsin-EDTA were obtained from Invitrogen (Thermo Fisher Scientific). Pyrrolidine dithiocarbamate (PDTC) and inhibitors were obtained from Sigma-Aldrich (St Louis, MO, USA). All other chemicals and reagents were of analytical grade and were obtained from commercial sources.

Male Sprague Dawley rats were purchased from Xinxiang Medical University, Henan, China. Animal experiments were approved by the Xinxiang Medical University institutional animal care and use committee and performed according to the guidelines for the care and use of laboratory animals.

\section{Preparation of FA-conjugated pullulan acetate NPs and FPA/EPI NPs}

First, FA-conjugated pullulan acetate was synthesized and the corresponding NPs were prepared by the dialysis method, as described in our previous publication. ${ }^{20}$

For EPI loading, typically, EPI (10 mg) was neutralized with $2 \mathrm{~mol}$ of excess triethylamine in $2 \mathrm{~mL}$ of dimethyl sulfoxide (DMSO). Subsequently, the EPI solution was added into $8 \mathrm{~mL}$ of a DMSO solution (DMSO: $\mathrm{H}_{2} \mathrm{O}=9: 1$ ) of FA-conjugated pullulan acetate $(100 \mathrm{mg}$, the degrees of substitution for acetyl groups and FA groups were $86.7 \%$ and $23.5 \%$, respectively) and mixed by vortexing for $10 \mathrm{~min}$. The mixture was transferred for dialysis (molecular weight cutoff: $14,000 \mathrm{Da}$ ) against deionized water for $48 \mathrm{~h}$.

\section{Characteristics of NPs}

The particle size was determined by dynamic light scattering (Zetasizer 2000; Malvern Instruments Ltd, Malvern, UK), and the zeta potential was also measured using the same instrument. The morphology of NPs was observed using transmission electron microscopy (TEM) (Tecnai ${ }^{\mathrm{TM}}$ G2 F20; FEI, Eindhoven, the Netherlands). TEM was performed using a negative-staining method. A drop of the NP dispersion was spread on a copper grid coated with carbon film. After staining with $2 \%$ phosphotungstic acid solution, it was dried at room temperature for TEM.

The morphology of NPs was characterized by field emission scanning electron microscopy (FESEM) (Zeiss 77 Supra 40VP; Carl Zeiss Microscopy GmbH, Jena, Germany) at $5.0 \mathrm{kV}$ voltage. To prepare samples for FESEM observations, a drop of the particle suspension was placed on a grid or a stud, and the supernatant liquid was removed with a capillary after the particles were allowed to settle. The particles were then coated with platinum for $30 \mathrm{~s}$.

\section{$\mathrm{KC}$ isolation}

$\mathrm{KCs}$ were harvested from the livers of anesthetized (under general anesthesia with ether) fasting Sprague Dawley rats (250-300 g) using the isolation buffers described previously. ${ }^{24-26}$ After iv administration of sodium heparin (100-200 units), the livers were exsanguinated in situ by portal vein perfusion with $\mathrm{Ca}^{2+}$-free Hanks' solution ( $\mathrm{pH} 7.4$ ), at a constant rate of $10-20 \mathrm{~mL} / \mathrm{min}$, and subsequently incubated with fresh collagenase buffer at $37^{\circ} \mathrm{C}$ for $5 \mathrm{~min}$. The liver was then excised and transferred into precooled PBS at $4^{\circ} \mathrm{C}$; then the liver membrane was cut, the liver was shaken gently, and the liver tissue was homogenized using a glass needle. After filtering through a 200-mesh nylon filter, we obtained the filtrate, which was the cell suspension.

Hepatocytes and cell clumps were removed from the suspension by low-speed centrifugation ( $50 \times g, 5 \mathrm{~min})$. The remaining cells were suspended in $12 \mathrm{~mL}$ RPMI-1640, then transferred into another $50 \mathrm{~mL}$ centrifuge tube with $15 \mathrm{~mL}$ of $50 \%$ Percoll solution and overlaid with $20 \mathrm{~mL}$ of $25 \%$ Percoll solution, followed by centrifugation $(800 \times g$, $20 \mathrm{~min}, 4^{\circ} \mathrm{C}$ ). Finally, the supernatant was discarded, and the cell pellet was resuspended in culture medium (RPMI-1640 supplemented with $10 \% \mathrm{FBS}, 100 \mu \mathrm{g} / \mathrm{mL}$ streptomycin, and $100 \mathrm{U} / \mathrm{mL}$ penicillin) and seeded into culture dishes. Following incubation for $2 \mathrm{~h}$ in a humidified atmosphere of $95 \%$ air with carbon dioxide $\left(\mathrm{CO}_{2}\right)$ at $37^{\circ} \mathrm{C}$, the cells were gently washed with fresh culture medium. The medium was changed every 2-3 d during the subsequent culture period. After reaching $80 \%$ confluence, the cells were washed with PBS and subsequently detached by incubation with $0.25 \%$ trypsin, counted using a hemocytometer, and subcultured in new culture plates. 
To measure cell viability, the upper solution was discarded after centrifugation, and the remaining 50\% Percoll solution (12 mL) was diluted with $12 \mathrm{~mL}$ RPMI-1640 containing $5 \%$ FBS, then cells were centrifuged $(950 \times g)$ for $10 \mathrm{~min}$ at $4^{\circ} \mathrm{C}$. Cell viability was determined by the trypan blue dye exclusion method. The cellular precipitate was collected, medium containing antibiotics was added, and the mixture was transferred into a Petri dish coated with rat tail collagen. The medium was changed after $4 \mathrm{~h}$ incubation at $37^{\circ} \mathrm{C}$, and cells were counted microscopically.

\section{$\mathrm{KC}$ culture}

KCs were cultured in RPMI-1640 medium (Thermo Fisher Scientific) supplemented with 5\% FBS and antibiotics ( $1 \%$ penicillin/streptomycin) at $37^{\circ} \mathrm{C}$ using a humidified $5 \%$ $\mathrm{CO}_{2}$ incubator. After culture for $48 \mathrm{~h}$, cultures were digested with $0.25 \%$ trypsin/EDTA, and then reseeded at a density of $5 \times 10^{5}$ cells $/ \mathrm{mL}$ in 96-well plates (Costar; Corning Inc, Corning, NY, USA) or $5 \times 10^{4}$ cells $/ \mathrm{mL}$ in six-well plates. When the cells reached $\sim 80 \%$ confluence, the medium was aspirated, and cells were fed with culture medium containing $200 \mu \mathrm{L}$ endocytosis inhibitors.

\section{Cellular uptake assay}

The capacity for cellular internalization of FPA/EPI NPs was quantified by fluorometry. KCs were seeded at a density of $5 \times 10^{5}$ cells $/ \mathrm{mL}$ in 96 -well plates and incubated with an FPA/EPI NPs suspension in serum-free medium $(200 \mu \mathrm{g} / \mathrm{mL})$, washed three times with PBS ( $\mathrm{pH} 7.4)$, and then lysed by incubating them with $1 \mathrm{~mL}$ PBS and $200 \mu \mathrm{L}$ of $0.5 \%$ Triton $^{\mathrm{TM}}$ X-100 (Sigma-Aldrich) in $0.2 \mathrm{~N} \mathrm{NaOH}$. Cell-associated FPA/EPI NPs were quantified by analyzing the cell lysate in a microplate reader $(\lambda \mathrm{ex}=485 \mathrm{~nm}, \lambda \mathrm{em}=538 \mathrm{~nm})$ calibrated with standard solutions containing $1-32.0 \mu \mathrm{g} / \mathrm{mL}$ of FPA/EPI NPs in cell lysate solution $\left(2 \times 10^{5}\right.$ untreated KCs lysed in $1 \mathrm{~mL}$ of Triton $\mathrm{X}-100$ solution). Uptake was expressed as the optical density (OD) of FPA/EPI NPs.

To study the effect of compound inhibitors on the uptake of NPs, cells were preincubated with the following inhibitors based on previous studies, ${ }^{27,28}$ at concentrations that were nontoxic to the cells: $7 \mu \mathrm{g} / \mathrm{mL}$ of chlorpromazine (CPZ; SigmaAldrich), to inhibit clathrin-mediated endocytosis; $50 \mu \mathrm{g} / \mathrm{mL}$ of nystatin (NY; Sigma-Aldrich) to inhibit caveolae-mediated endocytosis; $10 \mu \mathrm{g} / \mathrm{mL}$ of colchicine (Col; Sigma-Aldrich) to inhibit pinocytosis; and $50 \mu \mathrm{M}$ amiloride (AMR; SigmaAldrich), to inhibit macropinocytosis. At the same time, a NF-kB signal pathway inhibitor, PDTC (Sigma-Aldrich) was added at $10 \mu \mathrm{M} / \mathrm{mL}$ to inhibit the inflammatory effect, ${ }^{29}$ thus inhibiting the endocytosis of FPA/EPI NPs. Following preincubation for $24 \mathrm{~h}$ at $37^{\circ} \mathrm{C}$, the inhibitor solutions were removed, and freshly prepared FPA/EPI NPs, in medium containing inhibitors at the same concentrations, were added and further incubated for another $2 \mathrm{~h}$. Subsequently, the cells were washed as described previously. The group treated with FPA/EPI NPs but without inhibitor treatment was used as control, and its uptake was expressed as 100\%. Percentage uptake was calculated using the following formula:

$$
\text { Uptake } \%=(\text { OD }[\text { test }] / \text { OD }[\text { control }]) \times 100 \% \text {. }
$$

The dose- and time-dependent cellular uptake effect of inhibitors on FPA/EPI NPs was also determined. Cells were incubated with the following inhibitors individually: 0.35 , 3.5, and $35 \mu \mathrm{g} / \mathrm{mL}$ of CPZ; $1.25,12.5$, and $125 \mu \mathrm{g} / \mathrm{mL} \mathrm{NY}$; $0.25,2.5$, and $25 \mu \mathrm{g} / \mathrm{mL}$ Col; and 2.5, 25, and $250 \mu \mathrm{M}$ AMR; and $0.5,5$, and $50 \mu \mathrm{M}$ of PDTC. The preincubation time was $24 \mathrm{~h}$. To study the time course of the effect of various inhibitors on the uptake of NPs, cells were incubated with the following inhibitors individually: $7 \mu \mathrm{g} / \mathrm{mL} \mathrm{CPZ,} 50 \mu \mathrm{g} / \mathrm{mL}$ $\mathrm{NY}, 10 \mu \mathrm{g} / \mathrm{mL}$ Col, $50 \mu \mathrm{M}$ AMR, and $10 \mu \mathrm{M}$ of PDTC. The preincubation times were 2,6 , or $18 \mathrm{~h}$. The subsequent procedure was as described earlier.

\section{Cytokine analysis}

The culture supernatant was collected and stored at $-20^{\circ} \mathrm{C}$ until analysis. The level of the three proinflammatory cytokines (TNF- $\alpha$, IL-1 $\beta$, and IL-6) was determined in culture supernatants by bead-based multiplex flow cytometry using the AimPlex ${ }^{\circledR}$ human custom 3-plexkit (T3C035536; Beijing Quantobio Biotechnology Co Ltd, Beijing, China). In the AimPlex multiplex assay, multiple bead populations differentiated by size and different levels of fluorescence intensity - are coated with capture antibodies specific for different proinflammatory mediators. The AimPlex multiplex kit was used according to the manufacturer's instruction manual. The assay procedure consists of a 60 min antigen and capture antibody-conjugated bead incubation step, a 30 min biotinylated antibody incubation and detection step, and a 20 min streptavidin-phycoerythrin incubation step. The samples were placed on the flow cytometer (NovoCyte D1040; ACEA Biosciences, San Diego, CA, USA) to measure the fluorescence signal of the sample beads, and the results were analyzed using the FCAP Array software V3.0 (BD Biosciences, San Jose, CA, USA). The OD values and cytokine concentrations in the culture supernatant were expressed as the mean values \pm SDs. The inhibition percentage was calculated using the following formula: $(1-$ OD $[$ test $] / O D$ [control] $) \times 100 \%$. 


\section{Statistical analysis}

Averages and SDs (mean \pm SD) were calculated for each experimental group. Data were analyzed by one-way analysis of variance, and $P$-values $<0.05$ were considered significant.

\section{Results}

\section{Culture of KCs}

After differential centrifugation, nonparenchymal hepatocyterich cell fractions were obtained and cultured in tissue culture plates. After incubation for $4 \mathrm{~h}$, the cells became attached to the plate surface. The plates were rinsed with PBS to remove nonadherent cells, and the attached KCs were selectively harvested with good cell viability $(\sim 90 \%)$. KCs were identified by ink phagocytosis and immunofluorescence (Figure S1). The freshly isolated cells had a ball-like shape when viewed under a light microscope (Figure 1A). As culture time increased, they became larger, were more prominent, and exhibited an irregular outline (Figure 1B). Cells in mitosis were occasionally observed under a phase contrast microscope (Figure 1C).

\section{Characterizations of FPA/EPI NPs}

The self-assembled NPs were prepared by the dialysis method, which is a rapid and simple preparation method. The morphological characteristics of the self-assembled NPs could be observed by TEM (Figure 2A) and SEM (Figure 2B). FPA/EPI NPs were spherical in shape and almost uniformly sized. There was a clear hydrophobic nucleus in the middle of the NPs, and the surface was rough, as observed in our previous study. ${ }^{20}$

\section{Time-dependent effects on FPA/EPI NP uptake}

As shown in Figure 3, inhibitors had an obvious effect, with a statistically significant difference in uptake percentage ( $P<0.05$ or $P<0.01$ ), with NY, AMR, and Col showing timedependent inhibition effects. CPZ had the strongest effect at $2 \mathrm{~h}$, with an uptake percentage rate of $<40 \%$, and $\mathrm{NY}$ had the strongest effect at 6 and $18 \mathrm{~h}$. Therefore, we concluded that clathrin- and caveolae-mediated endocytosis were the most prominent pathways. As shown in Table 1, TNF- $\alpha$ levels in KCs decreased, while the other two proinflammatory mediators (IL-1 $\beta$ and IL-6), as determined by bead-based multiplex flow cytometry, did not change significantly. Col showed an obvious time-dependent inhibitory effect on TNF- $\alpha(P<0.05)$.

\section{Inhibitory effect of compound inhibitors}

The compound inhibitors showed an inhibitory effect on the uptake of FPA/EPI NPs by KCs. The uptake percentage in the presence of PDTC was $25.40 \%$, and PDTC + NY had the strongest effect, with an uptake rate of $14.62 \%$, while the uptake rates in the presence of PDTC + AMR, PDTC + CPZ and PDTC + Col were 20.19\%, 22.78\%, and 29.37\%, respectively (Figure 4). As shown in Table 2, the levels of the three different proinflammatory mediators (TNF- $\alpha$, IL-1 $\beta$, and IL-6) - as determined by enzyme-linked immunosorbent assay (ELISA) - changed significantly. TNF- $\alpha$ was inhibitied significantly by PDTC + AMR $(P<0.05$, $\mathrm{n}=5)$ and other inhibitors $(P<0.05, \mathrm{n}=5)$, whereas IL- $1 \beta$ and IL-6 were decreased less. The result showed that caveolaemediated pinocytosis is the main route of entry, although all four pathways were involved, and that the NF-kB signal pathway was very important.

\section{Dose-dependent effect of compound inhibitors}

To investigate the dose-dependent effect of various inhibitors on the uptake of FPA/EPI NPs, four endocytosis inhibitors together with PDTC at low, medium, or high doses (1-fold, 10-fold, and 100-fold) were preincubated with KCs. Compound inhibitors showed a much better uptake inhibition effect than PDTC alone; above all, PDTC + NY, PDTC + AMR, and PDTC + CPZ showed a dose-dependent effect, and PDTC $+\mathrm{CPZ}$ was the strongest inhibitor, with an uptake percentage of 35.75\% (Figure 5). As shown in Figure 6, the
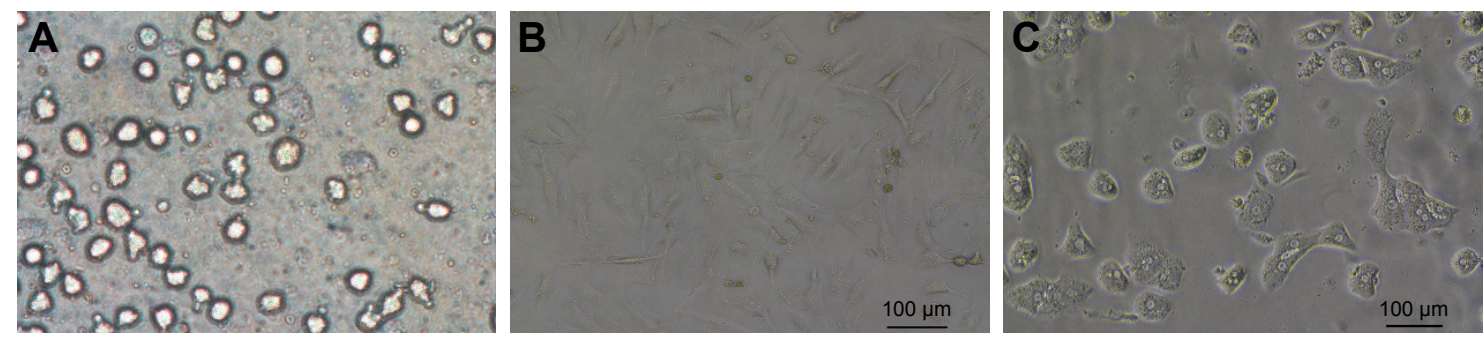

Figure I Photomicrographs of KCs in culture.

Notes: (A) Freshly isolated cells; (B) KCs cultured for 6 days; (C) cells in mitosis.

Abbreviation: KCs, Kupffer cells. 

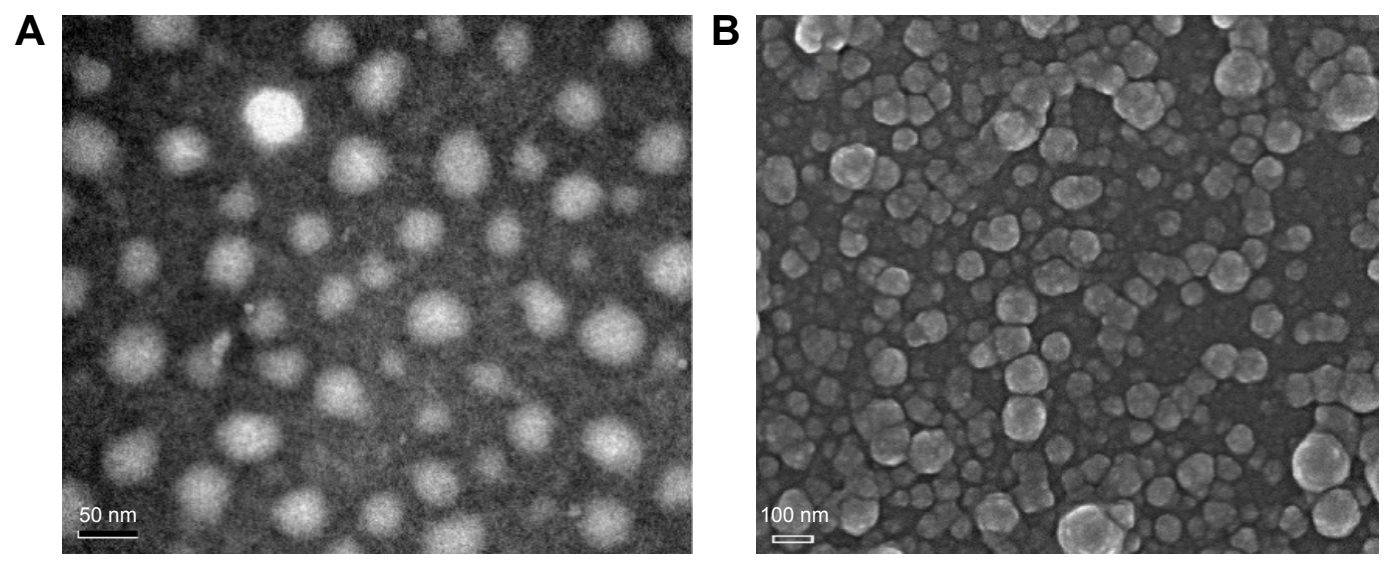

Figure 2 Morphology of FPA/EPI NPs prepared by the dialysis method.

Notes: (A) TEM images of FPA/EPI NPs. (B) SEM images of FPA/EPI NPs.

Abbreviations: FPA/EPI NPs, epirubicin-loaded folic acid-conjugated pullulan acetate nanoparticles; SEM, scanning electron microscopy; TEM, transmission electron microscopy.

three different proinflammatory mediators (TNF- $\alpha$, IL-1 $\beta$, and IL-6) - determined by ELISA - changed significantly. The compound inhibitors had a dose-dependent effect, and the inhibition effect of the high dose was almost the same as in the medium-dose groups. TNF- $\alpha$ was most markedly inhibited (Figure 6A) (with percentage inhibition ranging from $33.11 \%$ to $64.34 \%$ ), followed by IL-1 $\beta$ (with percentage inhibition from $26.43 \%$ to $59.78 \%$ ) (Figure 6B), and finally, IL-6 showed the least inhibition, with percentage inhibition from $4.06 \%$ to $33.95 \%$ (Figure $6 \mathrm{C}$ ). The results were consistent with the results of the earlier tests. This demonstrated once again that although all four pathways are involved, clathrin-mediated pinocytosis is the main internalization route and that the NF-kB signal pathway is very important.

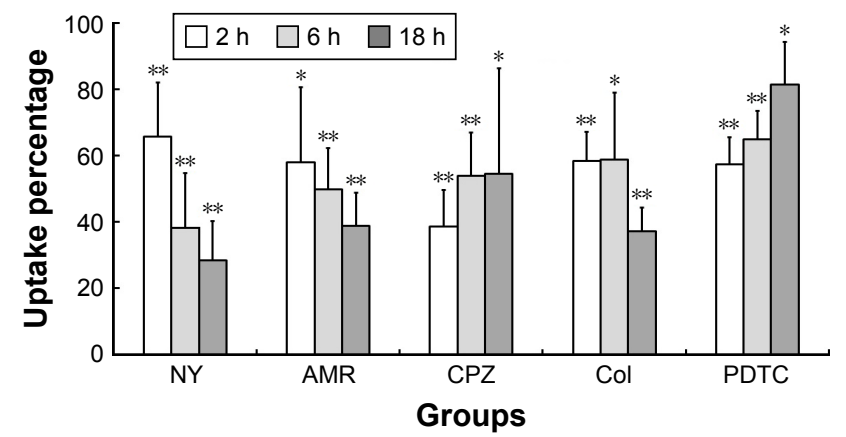

Figure 3 Time-dependent uptake of FPA/EPI NPs by KCs.

Notes: KCs were incubated with different endocytosis inhibitors for 2, 6, or $18 \mathrm{~h}$ prior to addition of $200 \mu \mathrm{g} / \mathrm{mL}$ FPA/EPI NPs, and the uptake percentage was quantified by fluorometry compared with untreated controls. Data are represented as mean \pm SD of five determinations. ${ }^{*} P<0.05$, $* * P<0.01$ compared to respective controls. Abbreviations: AMR, amiloride; Col, colchicine; CPZ, chlorpromazine; FPA/EPI NPs, epirubicin-loaded folic acid-conjugated pullulan acetate nanoparticles; KCs, Kupffer cells; NY, nystatin; PDTC, pyrrolidine dithiocarbamate.

\section{Discussion}

When NPs are administered into the blood, they are probably opsonized and cleared by macrophages. One of the predominant macrophage populations of the liver is constituted by the KCs. ${ }^{30}$ It is known that NPs can cross the cell membrane, but the mechanism of internalization is currently under debate. ${ }^{31}$ The endocytic pathway of FPA/EPI NPs in $\mathrm{KCs}$ has been first characterized in our study. CPZ and NY had the strongest inhibitory effect; therefore, clathrin- and caveolae-mediated endocytosis may be involved in the

Table I Time-dependent effect of different inhibitors on cytokine production by KCs

\begin{tabular}{llll}
\hline Groups & TNF- $\alpha, \mathrm{pg} / \mathrm{mL}$ & IL-I $\beta, \mathrm{pg} / \mathrm{mL}$ & IL-6, pg/mL \\
\hline Control & $2.20 \pm 0.20$ & $5.03 \pm 0.91$ & $7.73 \pm 1.23$ \\
NY I8 h & $1.87 \pm 0.15^{*}$ & $4.48 \pm 0.78$ & $7.51 \pm 1.49$ \\
NY 6 h & $1.87 \pm 0.25$ & $4.97 \pm 0.97$ & $6.94 \pm 0.89$ \\
NY 2 h & $1.96 \pm 0.28$ & $4.27 \pm 0.94$ & $6.69 \pm 1.39$ \\
AMR I8 h & $2.10 \pm 0.27$ & $4.93 \pm 0.73$ & $7.87 \pm 0.87$ \\
AMR 6 h & $1.68 \pm 0.56$ & $4.48 \pm 1.35$ & $7.25 \pm 1.18$ \\
AMR 2 h & $1.92 \pm 0.29$ & $4.59 \pm 1.16$ & $6.94 \pm 1.02$ \\
CPZ I8 h & $1.82 \pm 0.11 * *$ & $3.80 \pm 1.02$ & $6.43 \pm 1.44$ \\
CPZ 6 h & $2.13 \pm 0.32$ & $4.55 \pm 2.19$ & $6.14 \pm 0.33^{*}$ \\
CPZ 2 h & $1.92 \pm 0.26$ & $4.28 \pm 0.29$ & $7.45 \pm 0.56$ \\
Col 18 h & $1.68 \pm 0.36 *$ & $3.88 \pm 0.76$ & $6.53 \pm 0.95$ \\
Col 6 h & $1.68 \pm 0.30 *$ & $4.27 \pm 1.39$ & $6.74 \pm 1.59$ \\
Col 2 h & $1.74 \pm 0.21 *$ & $4.43 \pm 0.99$ & $6.21 \pm 1.23$ \\
PDTC 18 h & $1.85 \pm 0.25$ & $3.62 \pm 1.10$ & $5.82 \pm 0.09 *$ \\
PDTC 6 h & $1.73 \pm 0.23 *$ & $4.02 \pm 0.48$ & $7.06 \pm 1.59$ \\
PDTC 2 h & $1.88 \pm 0.21$ & $4.38 \pm 1.10$ & $5.20 \pm 0.80^{* *}$ \\
\hline
\end{tabular}

Notes: Values are presented as mean $\pm S D(n=5) . * P<0.05 ; * * P<0.01$ vs control. Abbreviations: AMR, amiloride; Col, colchicine; CPZ, chlorpromazine; IL, interleukin; KCs, Kupffer cells; NY, nystatin; PDTC, pyrrolidine dithiocarbamate; TNF- $\alpha$, tumor necrosis factor. 


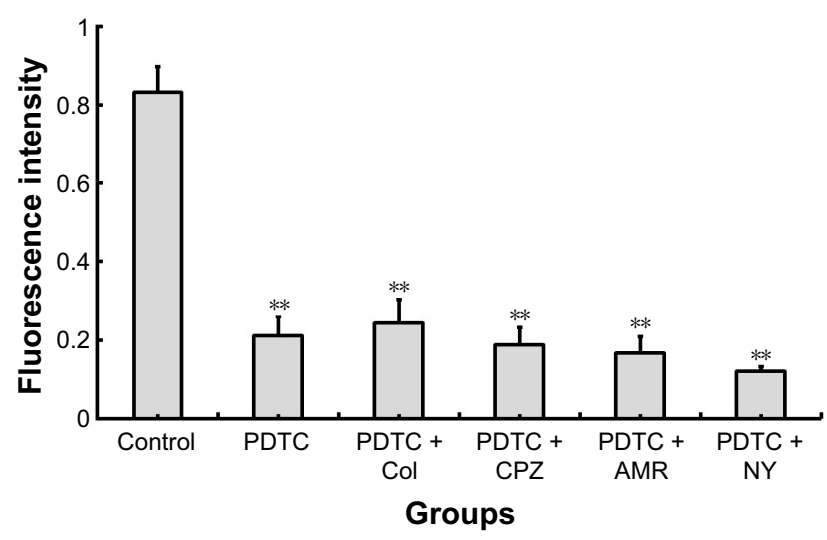

Figure 4 Compound inhibition of FPA/EPI NP uptake by KCs.

Notes: KCs were incubated with different compound endocytosis inhibitors for $24 \mathrm{~h}$ prior to addition of $200 \mu \mathrm{g} / \mathrm{mL}$ FPA/EPI NPs, and the nanoparticle uptake was quantified by fluorometry compared with untreated controls. Data are represented as mean $\pm S D$ of five determinations. $* * P<0.0$ l compared to respective controls. Abbreviations: AMR, amiloride; Col, colchicine; CPZ, chlorpromazine; FPA/EPI NPs, epirubicin-loaded folic acid-conjugated pullulan acetate nanoparticles; KCs, Kupffer cells; NY, nystatin; PDTC, pyrrolidine dithiocarbamate.

internalization of FPA/EPI NPs. Inhibitors had an obvious effect $(P<0.05$ or $P<0.01)$; NY and AMR showed a timedependent effect, while NY, AMR, CPZ, and Col showed dose-dependent effects. $\mathrm{CPZ}$ is a cationic amphipathic drug that relocates clathrin and adaptor protein complex-2 from the plasma membrane to the endosomal membrane..$^{32}$ The lipid raft inhibitor NY is used to block endocytosis that is mediated by caveolae. In our study, a combined mechanism, rather than a dominant mechanism, was determined to be involved in the cellular uptake of NPs and in cytokine production by macrophages following exposure to NPs. Clathrin- and caveolae-mediated endocytosis routes were the main pathways for the entry of FPANs into KCs. In our in vitro study, ${ }_{18}^{18}$ the cellular uptake and cellular internalization mechanisms of FPA/EPI NPs in HeLa (high expression of FR), MCF-7 (relatively high expression of FR), and HepG2 (low or no

Table 2 Inhibitory effect of compound inhibitors on cytokine production by KCs

\begin{tabular}{llll}
\hline Groups & TNF- $\alpha, \mathrm{pg} / \mathrm{mL}$ & $\mathrm{IL}-\mathrm{I} \beta, \mathrm{pg} / \mathrm{mL}$ & $\mathrm{IL}-6, \mathrm{pg} / \mathrm{mL}$ \\
\hline Control & $6.67 \pm 1.32$ & $5.07 \pm 0.8 \mathrm{I}$ & $9.54 \pm 0.88$ \\
PDTC & $2.53 \pm 0.37^{* *}$ & $5.66 \pm 0.99$ & $8.30 \pm 1.48$ \\
PDTC + Col & $2.57 \pm 0.22^{* *}$ & $5.12 \pm 0.99$ & $8.01 \pm 0.67$ \\
PDTC + CPZ & $3.03 \pm 0.2 I^{* *}$ & $6.61 \pm 0.38^{*}$ & $9.24 \pm 0.51$ \\
PDTC + AMR & $3.21 \pm 0.66^{*}$ & $7.56 \pm 0.77^{*}$ & $8.86 \pm 1.19$ \\
PDTC + NY & $2.69 \pm 0.62^{* *}$ & $7.58 \pm 0.84^{*}$ & $8.55 \pm 0.87$ \\
\hline
\end{tabular}

Notes: Time-dependent effects of different inhibitors on cytokine production by KCs. Data are represented as mean \pm SD of five determinations. $* P<0.05, * * P<0.01$ compared to control.

Abbreviations: AMR, amiloride; Col, colchicine; CPZ, chlorpromazine; IL, interleukin; KCs, Kupffer cells; NY, nystatin; PDTC, pyrrolidine dithiocarbamate; TNF- $\alpha$, tumor necrosis factor.

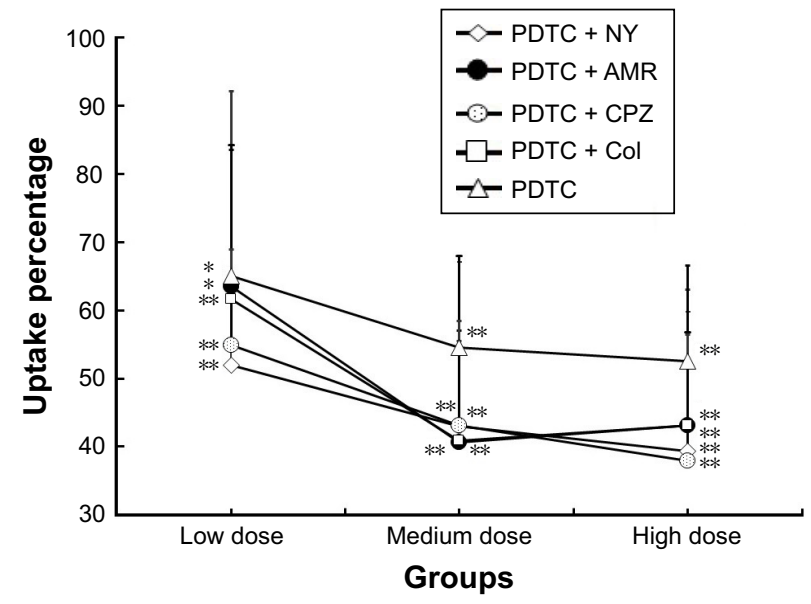

Figure 5 Dose-dependent uptake of FPA/EPI NPs by KCs.

Notes: KCs were incubated with different endocytosis inhibitors for $24 \mathrm{~h}$ prior to addition of $200 \mu \mathrm{g} / \mathrm{mL}$ FPA/EPI NPs, and the uptake was quantified by fluorometry compared with untreated controls. Data are represented as mean \pm SD of five determinations. $* P<0.05$, $* * P<0.01$ compared to respective controls.

Abbreviations: AMR, amiloride; Col, colchicine; CPZ, chlorpromazine; FPA/EPI NPs, epirubicin-loaded folic acid-conjugated pullulan acetate nanoparticles; KCs, Kupffer cells; NY, nystatin; PDTC, pyrrolidine dithiocarbamate.

expression of FR) cells were quantitatively detected by the fluorescence method. In the FR-overexpressing HeLa cells, the internalization of FPA/EPI NPs was significantly more efficient than that in other cancer cells. Apart from FR-mediated endocytosis, clathrin-mediated endocytosis and cellular macropinocytosis were involved in the cellular internalizations of FPA/EPI NPs in HeLa cells. On the basis of the endocytosis pathways in cancer cells and $\mathrm{KCs}$, it is possible to use caveolae-mediated endocytosis inhibitors to reduce the elimination of FPA/EPI NPs by KCs without affecting uptake by cancer cells. Sahay et $\mathrm{al}^{1}$ classify the various mechanisms of endocytosis available to nanomedicines, including phagocytosis and pinocytosis. The pathways of their entry depend on the cell type, as well as the particle size, shape, material composition, surface chemistry, and/or charge for utilization. Most nanomaterials have been shown to exploit more than one pathway to gain cellular entry. Therefore, in future, each study should use multiple cell types, utilize homogeneous NPs, and employ multiple tools to dissect trafficking at the same time. Another challenge is that these studies are in vitro studies, which should be verified by in vivo studies.

There was a significant increase in the levels of proinflammatory cytokines (IL-1, IL-6, and TNF- $\alpha$ ) during the uptake of NPs by KCs, and the inhibitors decreased the level of these cytokines and the quantity of endocytosed NPs. Yen et $\mathrm{a}^{133}$ have shown that GNPs upregulate the expression of proinflammatory genes (IL-1, IL-6, and TNF- $\alpha$ ) in the 


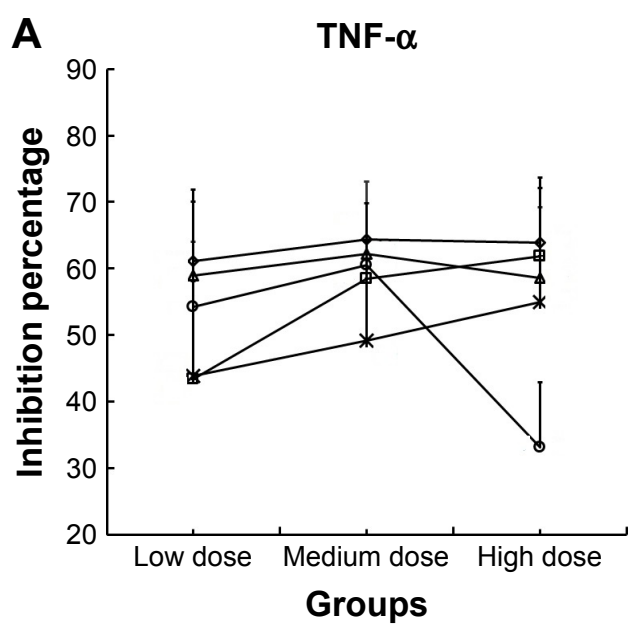

C

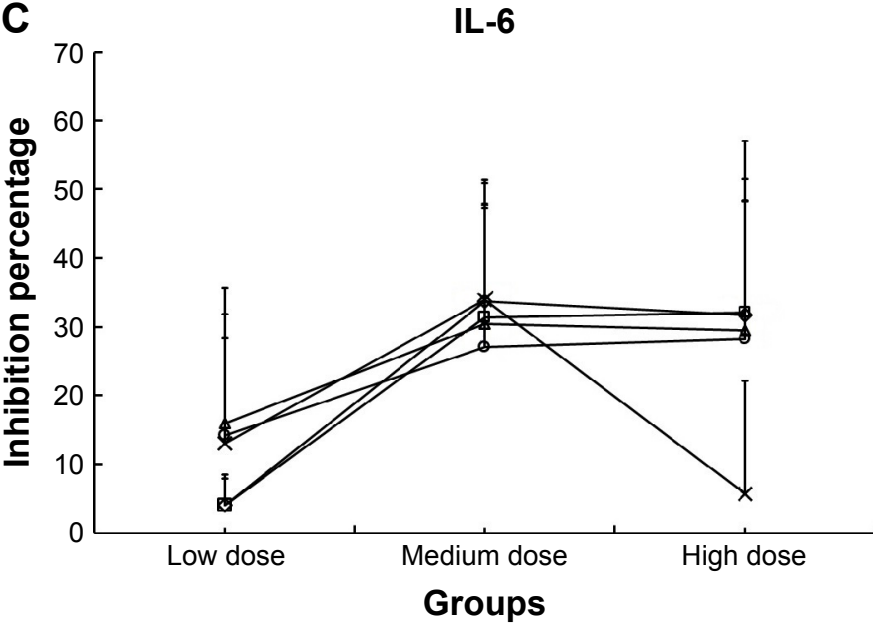

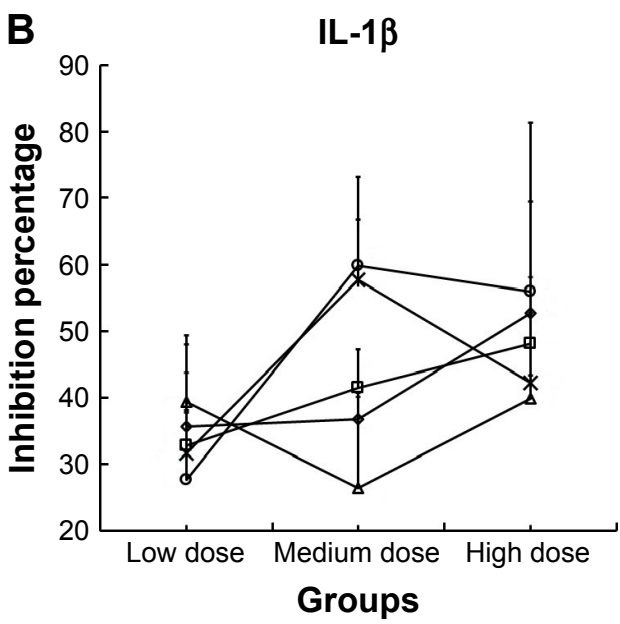

IL-6

PDTC + NY E PDTC + AMR

PDTC + CPZ

* PDTC + Col

PDTC

Figure 6 Dose-dependent inhibition of KC cytokines by compound inhibitors.

Notes: (A) The dose-dependent inhibition effect of compound inhibitors on cytokine TNF- $\alpha$. (B) The dose-dependent inhibition effect of compound inhibitors on cytokine IL-I $\beta$. (C) The dose-dependent inhibition effect of compound inhibitors on cytokine IL-6. KCs were incubated with different endocytosis compound inhibitors for $24 \mathrm{~h}$ prior to addition of $200 \mu \mathrm{g} / \mathrm{mL}$ FPA/EPI NPs, and the cytokine inhibition was quantified by fluorometry compared with untreated controls. Data are represented as mean \pm SD of five determinations.

Abbreviations: AMR, amiloride; Col, colchicine; CPZ, chlorpromazine; FPA/EPI NPs, epirubicin-loaded folic acid-conjugated pullulan acetate nanoparticles; IL, interleukin; KCs, Kupffer cells; NY, nystatin; PDTC, pyrrolidine dithiocarbamate; TNF- $\alpha$, tumor necrosis factor.

liver. As shown in Table 2 and Figure 6, TNF- $\alpha$ production by KCs was obviously inhibited by these inhibitors, while the other two proinflammatory mediators (IL-1 $\beta$ and IL-6), determined by bead-based multiplex flow cytometry, did not change significantly. TNF- $\alpha$ is the earliest and most important inflammatory mediator in the process of inflammation. Consequently, it is markedly changed earlier than the other two cytokines related to the NF-kB signal pathway. Stępkowski et $\mathrm{al}^{34}$ indicated that the NF-kB-mediated cellular response to AgNPs was cell type specific and related to the basal activity of NF-kB. PDTC, an NF-kB inhibitor, inhibits endocytosis by inhibiting proinflammatory effects. As shown in Figures 4 and 5, compound inhibitors had a greater effect than PDTC alone; most notably, PDTC + NY, PDTC + AMR, and PDTC
+ CPZ showed dose-dependent effects, while PDTC + CPZ had the strongest inhibitory effect. When the inhibitor dosage was 10 times (high-dose group) the medium dose, the inhibitory effect of the cytokines did not increase any further. The medium dose showed the maximal effect. The concentrations of the three different proinflammatory mediators (TNF- $\alpha$, IL-1 $\beta$, and IL-6), as determined by ELISA, all changed significantly (Figure 6). TNF- $\alpha$ showed the strongest inhibition, and the results were in agreement with the earlier results.

Our experiments showed that the uptake of NPs by KCs was closely correlated with inflammation; three of the cytokines were related to NF-kB signaling pathways. PDTC had a dose-dependent inhibition effect on the uptake of NPs through its inhibition of NF-kB activation to prevent the expression of 
adhesion molecules and cytokines. Secondly, uptake pathway inhibitors in KCs reduced not only the uptake of NPs by KCs but also the level of cytokines. Our previous results ${ }^{20}$ showed that the amount of EPI encapsulated in FPANs distributed in the liver is 5.7-11.6 times that of the free drug in the cervical cancer transplantation tumor animal models. The in vivo biodistribution of FPA/EPI NPs will be clarified in our next studies, in which we aim to confirm that FPA/EPI NPs could escape liver $\mathrm{KC}$ endocytosis in mice pretreated with inhibitors.

In summary, inhibitors of NF-kB signaling or endocytosis pathways could inhibit the FPA/EPI NPs endocytosis by liver $\mathrm{KCs}$ in vitro. This has great potential to prolong the circulation time of FPA/EPI NPs in the body, thus improving the targeted therapy effect.

\section{Conclusion}

The cellular uptake of FPANs was dependent on both dose and time. Two coexisting uptake mechanisms were involved in NP uptake by KCs, clathrin- and caveolae-mediated endocytosis being the main routes of entry. Our findings suggest that such an endocytosis inhibition strategy has great potential for improving the therapeutic effects and reducing the toxicity of NDDSs in the treatment of cancer.

\section{Acknowledgments}

This work was financially supported by the National Natural Science Foundation of China (grant number 81301322, 81401519), Beijing Natural Science Foundation (grant number 7132064) and Beijing Municipal Science \& Technology Commission (grant number Z151100003815016, Z141107002514146). Hongli Chen is the co-first author.

\section{Disclosure}

The authors report no conflicts of interest in this work.

\section{References}

1. Sahay G, Alakhova DY, Kabanov AV. Endocytosis of nanomedicines. J Control Release. 2010;145(3):182-195.

2. Barua S, Mitragotri S. Challenges associated with penetration of nanoparticles across cell and tissue barriers: a review of current status and future prospects. Nano Today. 2014;9(2):223-243.

3. Igarashi E. Factors affecting toxicity and efficacy of polymeric nanomedicines. Toxicol Appl Pharmacol. 2008;229(1):121-134.

4. Bilzer M, Roggel F, Gerbes AL. Role of Kupffer cells in host defense and liver disease. Liver Int. 2006;26:1175-1186.

5. Morais T, Soares ME, Duarte JA, et al. Effect of surface coating on the biodistribution profile of gold nanoparticles in the rat. Eur J Pharm Biopharm. 2012;80(1):185-193.

6. Sadauskas E, Wallin H, Stoltenberg M, et al. Kupffer cells are central in the removal of nanoparticles from the organism. Part Fibre Toxicol. 2007;4:10.

7. Balasubramanian SK, Jittiwat J, Manikandan J, Ong CN, Yu LE, Ong WY. Biodistribution of gold nanoparticles and gene expression changes in the liver and spleen after intravenous administration in rats. Biomaterials. 2010;31(8):2034-2042.
8. Bartneck M, Warzecha KT, Tacke F. Therapeutic targeting of liver inflammation and fibrosis by nanomedicine. Hepatobiliary Surg Nutr. 2014;3(6):364-376.

9. Sanhai WR, Sakamoto JH, Canady R, et al. Seven challenges for nanomedicine. Nat Nanotechnol. 2008;3(5):242-244.

10. Yoo JW, Chambers E, Mitragotri S. Factors that control the circulation time of nanoparticles in blood: challenges, solutions and future prospects. Curr Pharm Des. 2010;16(21):2298-2307.

11. Sun L, Wu Q, Peng F, Liu L, Gong C. Strategies of polymeric nanoparticles for enhanced internalization in cancer therapy. Colloids Surf B Biointerfaces. 2015;135:56-72.

12. Wang H, Wu L, Reinhard BM. Scavenger receptor mediated endocytosis of silver nanoparticles into J774A.1 macrophages is heterogeneous. ACS Nano. 2012;6(8):7122-7132.

13. Zhu F, Yue W, Wang Y. The nuclear factor kappa B (NF- $\mathrm{BB})$ activation is required for phagocytosis of Staphylococcus aureus by RAW 264.7 cells. Exp Cell Res. 2014;327(2):256-263.

14. Khan HA, Abdelhalim MA, Alhomida AS, AI-Ayed MS. Effects of naked gold nanoparticles on proinflammatory cytokines mRNA expression in rat liver and kidney. Biomed Res Int. 2013;2013: 590730 .

15. Scherbart AM, Langer J, Bushmelev A, et al. Contrasting macrophage activation by fine and ultrafine titanium dioxide particles is associated with different uptake mechanisms. Part Fibre Toxicol. 2011;8:31.

16. Xu L, Bai Q, Zhang X, Yang H. Folate-mediated chemotherapy and diagnostics: an updated review and outlook. J Control Release. 2017; 252:73-82.

17. Duan S, Guo L, Shi D, Shang M, Meng D, Li Q. Development of a novel folate-modified nanobubbles with improved targeting ability to tumor cells. Ultrason Sonochem. 2017;37:235-243.

18. Chen HL, Jia YJ, Nan WB, et al. Cytotoxicity and cellular internalization of epirubicin-loaded folate-conjugated pullulan acetate nanoparticles in cancer cells. Sci Adv Mater. 2016;8(11):2029-2036.

19. Kim H, Jo A, Baek S, et al. Synergistically enhanced selective intracellular uptake of anticancer drug carrier comprising folic acid-conjugated hydrogels containing magnetite nanoparticles. Sci Rep. 2017;7:41090.

20. Tang H, Feng X, Zhang T, et al. Stability, pharmacokinetics, biodistribution and safety assessment of folate-conjugated pullulan acetate nanoparticles as cervical cancer targeted drug carriers. J Nanosci Nanotechnol. 2015;15(9):6405-6412.

21. Zhang HZ, Li XM, Gao FP, Liu LR, Zhou ZM, Zhang QQ. Preparation of folate-modified pullulan acetate nanoparticles for tumor-targeted drug delivery. Drug Deliv. 2010;17(1):48-57.

22. Ji J, Zuo P, Wang YL. Enhanced antiproliferative effect of carboplatin in cervical cancer cells utilizing folate-grafted polymeric nanoparticles. Nanoscale Res Lett. 2015;10(1):453.

23. Singh RS, Kaur N, Kennedy J. Pullulan and pullulan derivatives as promising biomolecules for drug and gene targeting. Carbohydr Polym. 2015;123:190-207.

24. Zeng WQ, Zhang JQ, Li Y, Yang K, Chen YP, Liu ZJ. A new method to isolate and culture rat Kupffer cells. PLoS One. 2013;8(8):e70832.

25. Thomas P, Hayashi H, Lazure D, et al. Inhibition of lipopolysaccharide activation of Kupffer cells by transition metals. J Surg Res. 2008; 148(2):116-120.

26. Naito M, Hasegawa G, Ebe Y, Yamamoto T. Differentiation and function of Kupffer cells. Med Electron Microsc. 2004;37(1):16-28.

27. Plummer EM, Manchester M. Endocytic uptake pathways utilized by CPMV nanoparticles. Mol Pharm. 2013;10(1):26-32.

28. Jiang L, Li X, Liu L, Zhang Q. Cellular uptake mechanism and intracellular fate of hydrophobically modified pullulan nanoparticles. Int J Nanomedicine. 2013;8:1825-1834.

29. Han SG, Newsome B, Hennig B. Titanium dioxide nanoparticles increase inflammatory responses in vascular endothelial cells. Toxicology. 2013; 306:1-8.

30. Roy R, Kumar S, Tripathi A, Das M, Dwivedi PD. Interactive threats of nanoparticles to the biological system. Immunol Lett. 2014;158(1-2): 79-87. 
31. Vilella A, Ruozi B, Belletti D, et al. Endocytosis of nanomedicines: the case of glycopeptide engineered PLGA nanoparticles. Pharmaceutics. 2015;7(2):74-89.

32. Wang LH, Rothberg KG, Anderson RG. Mis-assembly of clathrin lattices on endosomes reveals a regulatory switch for coated pit formation. J Cell Biol. 1993;123(5):1107-1117.
33. Yen HJ, Hsu SH, Tsai CL. Cytotoxicity and immunological response of gold and silver nanoparticles of different sizes. Small. 2009;5(13):1553-1561.

34. Stępkowski TM, Brzóska K, Kruszewski M. Silver nanoparticles induced changes in the expression of NF- $\kappa \mathrm{B}$ related genes are cell type specific and related to the basal activity of NF- $\mathrm{BB}$. Toxicol In Vitro. $2014 ; 28(4): 473-478$. 


\section{Supplementary material}
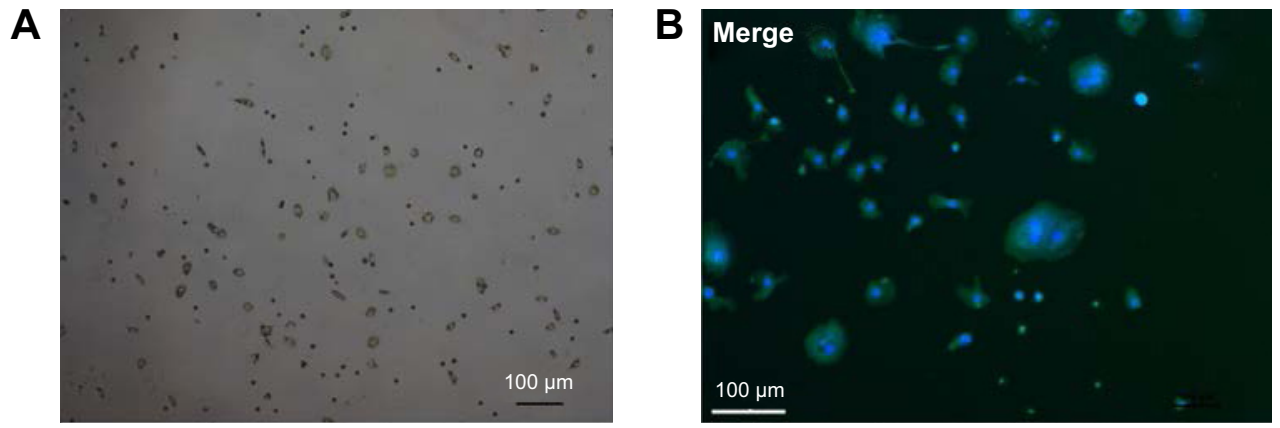

Figure SI Identification of KCs in culture.

Notes: (A) Ink phagocytosis experiment to confirm identification of KCs. (B) Immunofluorescence microscopy of KCs.

Abbreviation: $\mathrm{KCs}$, Kupffer cells.

\section{Publish your work in this journal}

The International Journal of Nanomedicine is an international, peerreviewed journal focusing on the application of nanotechnology in diagnostics, therapeutics, and drug delivery systems throughou the biomedical field. This journal is indexed on PubMed Central, MedLine, CAS, SciSearch $®$, Current Contents $\AA /$ Clinical Medicine,
Journal Citation Reports/Science Edition, EMBase, Scopus and the Elsevier Bibliographic databases. The manuscript management system is completely online and includes a very quick and fair peer-review system, which is all easy to use. Visit http://www.dovepress.com/ testimonials.php to read real quotes from published authors.

\footnotetext{
Submit your manuscript here: http://www.dovepress.com/international-journal-of-nanomedicine-journal
} 